\title{
Simple Synthesis and Luminescence Characteristics of PVP-Capped $\mathrm{GeO}_{2}$ Nanoparticles
}

\author{
Wei Wu, Xu Zou, Quanjun Li, Bingbing Liu, Bo Liu, Ran Liu, Dedi Liu, Zepeng Li, \\ Wen Cui, Zhaodong Liu, Dongmei Li, Tian Cui, and Guangtian Zou
}

State Key Laboratory of Superhard Materials, Jilin University, Changchun 130012, China

Correspondence should be addressed to Bingbing Liu, liubb@jlu.edu.cn

Received 30 April 2010; Accepted 28 July 2010

Academic Editor: Quanqin Dai

Copyright (c) 2011 Wei Wu et al. This is an open access article distributed under the Creative Commons Attribution License, which permits unrestricted use, distribution, and reproduction in any medium, provided the original work is properly cited.

\begin{abstract}
Polyvinylpyrrolidone (PVP)-capped rutile $\mathrm{GeO}_{2}$ nanoparticles were synthesized through a facile hydrothermal process. The obtained nanoparticles were characterized by X-ray diffraction (XRD), transmission electron microscopy (TEM), Fourier transform infrared spectroscopy (FTIR), thermo gravimetric analysis (TGA), and photoluminescence spectroscopy (PL). The capped $\mathrm{GeO}_{2}$ nanoparticles showed significantly enhanced luminescence properties compared with those of the uncapped ones. We attributed this result to the effect of reducing surface defects and enhancing the possibility of electron-hole recombination of the $\mathrm{GeO}_{2}$ nanoparticles by the PVP molecules. PVP-capped $\mathrm{GeO}_{2}$ nanoparticles have potential application in optical and electronic fields.
\end{abstract}

\section{Introduction}

Germanium oxide $\left(\mathrm{GeO}_{2}\right)$ is an important semiconductor material that has attracted much interest owing to its unique optical property and silica analogue $[1,2]$. Moreover, nanostructured $\mathrm{GeO}_{2}$ possesses the superior physical and chemical properties compared with its bulk counterparts. Nowadays, it is being widely used in optoelectronic devices, vacuum technology, and catalysis. For example, $\mathrm{GeO}_{2}$ nanowires were used in one-dimensional luminescence nanodevices by Sahnoun et al. [3]. And $\mathrm{GeO}_{2}$ nanotubes and nanorods as an important optical fibre material have been used in thermal vacuum test successfully by Jiang et al. [4].

It is well known that $\mathrm{GeO}_{2}$ forms in two stable crystalline structures at ambient temperature, the $\alpha$-quartz trigonal structure and the rutile tetragonal structure [5]. Over the past decades several properties of $\alpha$-quartz-type $\mathrm{GeO}_{2}$ have been investigated, for instance blue luminescence and dielectric properties. Compared with $\alpha$-quartz-type $\mathrm{GeO}_{2}$, rutile $\mathrm{GeO}_{2}$ has some unique properties, such as excellent transmissivity and green luminescence [6-8]. For these properties, rutile $\mathrm{GeO}_{2}$ has been considered as a potential material for luminescent device [9]. However, rutile $\mathrm{GeO}_{2}$ nanoparticles, like other semiconductor nanoparticles, have high surface energy, and they agglomerate or coalesce extremely quickly [10]. For this reason, many methods have been used to improve the stability of nanoparticles, such as changing of annealing temperature and doping of semiconductor and surfaces capped by various organic or inorganic layers, [11-13]. Among these methods, polymer capping as a newly chemical method has been developed to synthesize nanoparticles with high surface stability and also has significant influence on the morphology and optical properties of nanoparticles [14]. Moreover, compared with the aforementioned methods, it also has several other advantages, such as facile process and gentle reaction conditions However, the rutile $\mathrm{GeO}_{2}$ modified by PVP had never been synthesized successfully before. So this is a problem that should be solved quickly.

In this present work, we report, for the first time, the synthesis of PVP-capped rutile $\mathrm{GeO}_{2}$ nanocrystals through a simple hydrothermal process. PVP, which is a water soluble polymer, was used as capping polymer molecule to stabilize the $\mathrm{GeO}_{2}$ nanoparticles. Through the surface modification by PVP, highly monodisperse $\mathrm{GeO}_{2}$ nanoparticles were prepared, which exhibited highly chemical stability and 


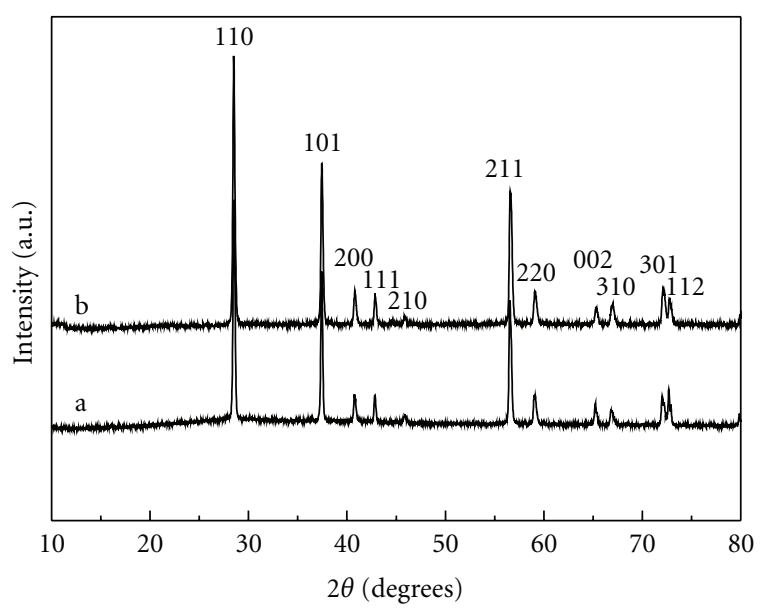

FIGURE 1: XRD pattern of the uncapped (a) and PVP-capped (b) $\mathrm{GeO}_{2}$ nanoparticles.

significantly enhanced luminescence. Our study provides a new hybrid material which has potential application in the field of nanoscale device.

\section{Experimental Section}

2.1. Material and Methods. We synthesize the samples through hydrothermal synthesis method. In a typical preparation process, $0.1 \mathrm{~g} \mathrm{GeO}_{2}$ was added in $15 \mathrm{~mL} \mathrm{HCl}$ aqueous solution $(\mathrm{PH}=1)$ in a Teflon-lined autoclave of $20 \mathrm{~mL}$ capacity. The autoclave was maintained at $180^{\circ} \mathrm{C}$ for 12 hours and then naturally cooled to room temperature. The obtained solution was discarded. The powder samples were then redispersed in distilled water in an ultrasonic bath. The centrifugation was repeated twice so as to remove the $\mathrm{HCl}$ residue. After that, the powder samples were dried in an oven at $60^{\circ} \mathrm{C}$ for 24 hours in air. Then the powder samples were added in a Teflon-lined autoclave of $20 \mathrm{ml}$ capacity again with $15 \mathrm{~mL}$ distilled water and $0.2 \mathrm{~g}$ PVP. The autoclave was maintained at $140^{\circ} \mathrm{C}$ for 6 hours and then cooled to room temperature naturally. The centrifugation was repeated twice so as to remove the aqueous solution PVP again. The powder samples were dried in an oven at $60^{\circ} \mathrm{C}$ for 24 hours in air. Then PVP-capped $\mathrm{GeO}_{2}$ was obtained. These powder samples were collected for further characterization. All the chemical reactants were analytical grade.

2.2. Characterizations Used. The as-prepared products were characterized using X-ray powder diffractometer (XRD, Rigaku, D/max-RA), transmission electron microscopy (TEM, HITACHI H-8100), Fourier transform infrared spectrometer (FTIR, NA-360), thermogravimetric analyzer (TGA, Perkin-Elmer), and Raman spectrometer (Renishaw 1000) using excitation wavelengths of $325 \mathrm{~nm}$ (He-Cd laser). Besides, the particle size and distribution were determined by measuring the maximum diameter of more than 100 particles on the TEM images.

\section{Results and Discussion}

3.1. XRD. Figure 1 shows a typical powder XRD pattern of uncapped (a) and PVP-capped (b) $\mathrm{GeO}_{2}$ nanocrystals prepared by the hydrothermal experiments. All the diffraction peaks could be indexed to rutile structure $\mathrm{GeO}_{2}$ with cell constants $\mathrm{a}_{0}=4.831 \AA$ and $\mathrm{c}_{0}=5.568 \AA$. These XRD peaks are in good agreement with those of the JCPDS card (no. 880285). It indicates that the obtained products are all of pure rutile phase $\mathrm{GeO}_{2}$. No obvious differences can be observed in the XRD patterns of the uncapped (Figure 1(a)) and PVPcapped (Figure 1(b)) $\mathrm{GeO}_{2}$ nanoparticles. This shows that PVP modification does not influence the structure of $\mathrm{GeO}_{2}$.

3.2. TEM. Figure 2 shows typical TEM images of the noncapped (Figure 2(a)) and PVP-capped (Figure 2(b)) $\mathrm{GeO}_{2}$ nanoparticles. We also provide the particle size and distribution histograms of the noncapped (Figure 3(a)) and PVP-capped (Figure 3(a)) $\mathrm{GeO}_{2}$ nanoparticles. Average sizes of the uncapped $\mathrm{GeO}_{2}$ are in the range of $700-800 \mathrm{~nm}$ estimated from TEM images (Figure 2(a)). The obvious aggregation of the uncapped $\mathrm{GeO}_{2}$ nanocrystals may be due to the high surface energy of the nanocrystals. From Figure 2(b), the PVP-capped $\mathrm{GeO}_{2}$ nanoparticles had the same size (about $500 \mathrm{~nm}$ ) compared with those without PVP modification. Moreover, we found that there were some smaller particles appeared in the TEM images. We thought that these small particles are also done when the $\mathrm{GeO}_{2}$ samples were uncapped with PVP. Because of the aggregation are much bigger than single particle, especially for the small size particle. When the small size is particle adsorbed on the aggregation, it is difficult to find it in the TEM images. Above all, from the TEM images, we could find that PVP plays an important role in monodispersion property of the $\mathrm{GeO}_{2}$ nanoparticles. In this process PVP lowered the surface energy of the nanocrystals, so capped $\mathrm{GeO}_{2}$ nanoparticles with monodispersion property were obtained as observed [15].

3.3. FTIR. The polymer capping of germanium oxide is confirmed through FTIR spectroscopy. Figure 4 shows typical FTIR spectra of $\mathrm{GeO}_{2}$ nanoparticles (Figure 4(a)) and the $\mathrm{GeO}_{2}$ nanoparticles modified with PVP (Figure 4(b)). The absorption bands around $3400 \mathrm{~cm}^{-1}$ observed in both spectra are attributed to $\mathrm{O}-\mathrm{H}$ stretching mode of water and hydroxyl. The band (Figure $4(\mathrm{a})$ ) at $\sim 550 \mathrm{~cm}^{-1}$ corresponds to $\mathrm{Ge}-\mathrm{OH}$ stretching motion, and the band at $\sim 899 \mathrm{~cm}^{-1}$ corresponds to $\mathrm{Ge}-\mathrm{O}-\mathrm{Ge}$ stretching motion. The two stretching vibration bands located at $\sim 550$ and $\sim 889 \mathrm{~cm}^{-1}$ are the characteristic peaks of $\mathrm{GeO}_{2}$ crystal [16]. The FTIR spectrum of PVP-capped $\mathrm{GeO}_{2}$ shows some remarkable spectral changes. The most prominent and informative bands in the spectrum of compounds occur in the high frequency range between 400 and $1900 \mathrm{~cm}^{-1}$. For example the band at $750 \mathrm{~cm}^{-1}$ replaces the dominant peak of $\mathrm{GeO}_{2}$, which corresponds to the Ge-O-C bending [17]. The most convincing evidence from FTIR spectra of the PVP-modified $\mathrm{GeO}_{2}$ is the absorption peaks at 1265 and $1628 \mathrm{~cm}^{-1}$ which 


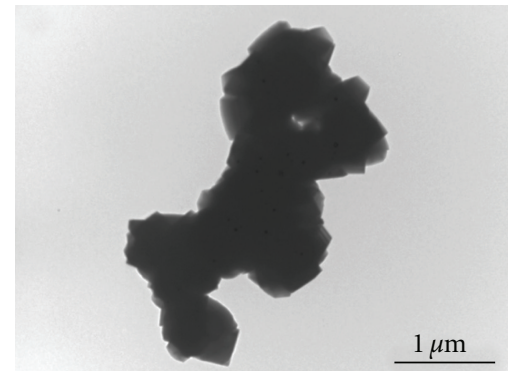

(a)

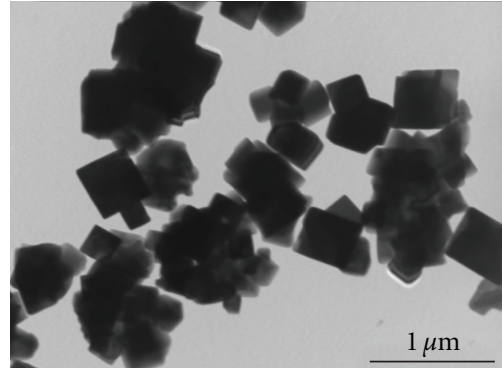

(b)

Figure 2: TEM images of the noncapped (a) and PVP-capped (b) $\mathrm{GeO}_{2}$ nanoparticles.

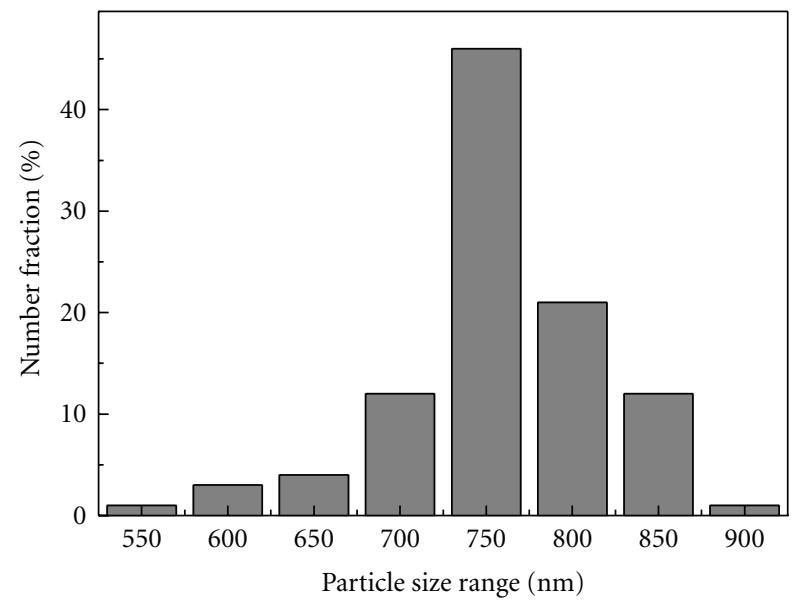

(a)

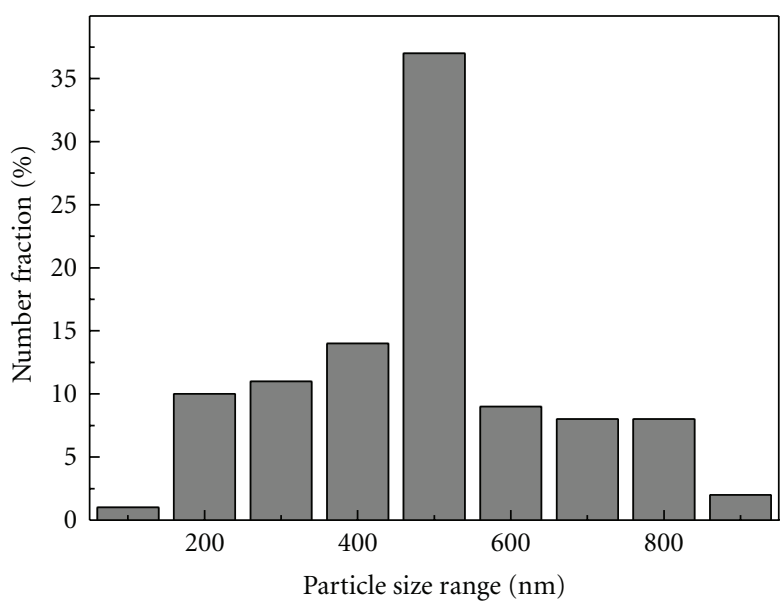

(b)

FIgure 3: Particle size distribution histograms of the noncapped (a) and PVP-capped (b) $\mathrm{GeO}_{2}$ nanoparticles.

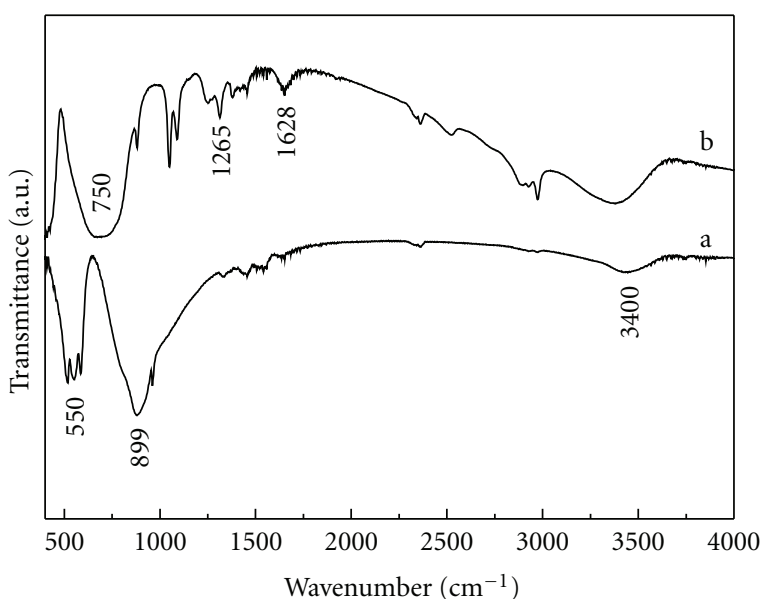

FIGURE 4: FTIR spectra of noncapped (a) and PVP-capped (b) $\mathrm{GeO}_{2}$ nanoparticles.

correspond to $\mathrm{C}-\mathrm{N}$ stretching motion and $\mathrm{C}=\mathrm{O}$ stretching motion of monomer for PVP, respectively [18-20]. The band at $\sim 680 \mathrm{~cm}^{-1}$ of Ge-O-C indicates that stable bonding exists between the $\mathrm{GeO}_{2}$ and some organic. Moreover the absorption peaks at 1265 and $1628 \mathrm{~cm}^{-1}$ occurred in Figure 4(b), this is due to the presence of PVP [21]. These previous discussions of the FTIR spectroscopy confirm that the surface of obtained $\mathrm{GeO}_{2}$ particles is modified with PVP successfully.

3.4. TGA. The TGA of PVP-capped $\mathrm{GeO}_{2}$ samples was carried out in an inert nitrogen atmosphere in the temperature range of $50 \sim 900^{\circ} \mathrm{C}$. The heating rate employed was $20^{\circ} \mathrm{C} / \mathrm{min}$. The obtained TGA curve is shown in Figure 5. A continuous weight loss in TGA from 500 to $700^{\circ} \mathrm{C}$ indicates PVP decomposition. This suggests that the PVP decomposition in $\mathrm{GeO}_{2}$ modified by PVP starts at a temperature much higher than its synthesized temperature $\left(\sim 150^{\circ} \mathrm{C}\right)$. These results indicate that the $\mathrm{GeO}_{2}$ nanoparticles were also successfully modified with PVP.

3.5. PL Spectra. The PL spectra were recorded with the excitation wavelength of $325 \mathrm{~nm}$. The PL spectra of the noncapped (Figure 6(a)) and PVP-capped (Figure 6(b)) $\mathrm{GeO}_{2}$ nanoparticles were shown in Figure 6. Both samples exhibited an emission band from 570 to $690 \mathrm{~nm}$, but the relative intensity varied. Contrasting the PL spectra of PVPcapped and uncapped $\mathrm{GeO}_{2}$ nanoparticles, it is obvious that 


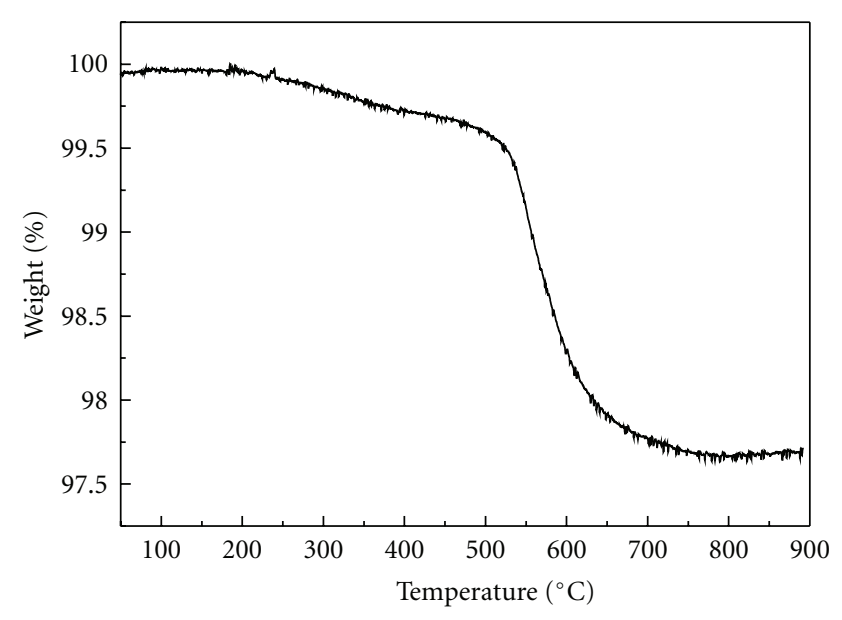

Figure 5: TGA curve for PVP-capped $\mathrm{GeO}_{2}$ taken in nitrogen atmosphere.

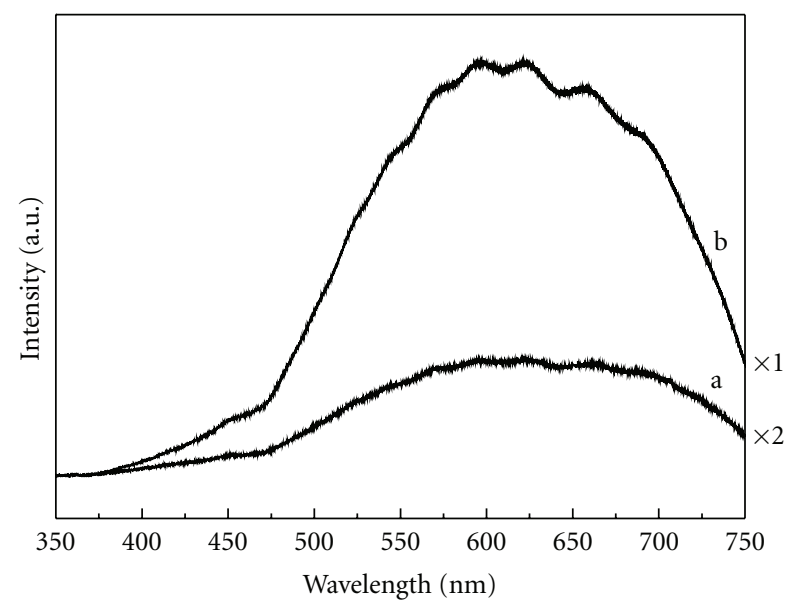

FIGURE 6: PL spectra of the noncapped (a) and PVP-capped (b) $\mathrm{GeO}_{2}$ nanoparticles.

the emission band of the uncapped particle is quite broad, and the PL intensity of capped nanoparticles is enhanced about one order of magnitude stronger than that of the noncapped ones. This result clearly justifies that the PVP as the capping agent can significantly enhance the PL intensity for $\mathrm{GeO}_{2}$ nanoparticles.

As it is known, the emission band was assigned to the surface trap-induced fluorescence, which involved the recombination of electrons trapped inside a germanium vacancy with a hole in the valence band of the $\mathrm{GeO}_{2}$ nanoparticles [22]. In our case, the enhanced luminescence properties of the capped $\mathrm{GeO}_{2}$ nanoparticles may be due to the PVP surface modification which may have the effect of minimizing surface defects and enhance the possibility of electron-hole recombination [23]. Besides PVP, other polymers, such as poly(ethylene glycol) (PEG), cetyltrimethylammonium bromide (CTAB), and oleic acid were also used. However, PVP was the effective polymer that could improve the luminescence properties of $\mathrm{GeO}_{2}$ nanoparticles obviously.

\section{Conclusion}

In summary, we have successfully synthesized monodisperse PVP-capped rutile $\mathrm{GeO}_{2}$ nanoparticles, via a facile hydrothermal process. This result indicates that PVP could control the morphology of rutile $\mathrm{GeO}_{2}$ effectively. In addition, the PL intensity of capped nanoparticles is enhanced about one order of magnitude stronger than that of the noncapped ones. We suggested that this performance improvement is due to the PVP surface modification which has the effect of minimizing surface defects and enhance the possibility of electron-hole recombination.

\section{Acknowledgments}

This paper was supported financially by the NSFC (10979001 and 20773043), the National Basic Research Program of China (2005CB724400), the Program for the Changiiang Scholar and Innovative Research Team in University (IRT0625), the Cheung Kong Scholars Programme of China, and the National Fund for Fostering Talents of Basic Science (J0730311).

\section{References}

[1] S. Grandi, P. Mustarelli, S. Agnello, M. Cannas, and A. Cannizzo, "Sol-gel $\mathrm{GeO}_{2}$-doped $\mathrm{SiO}_{2}$ glasses for optical applications," Journal of Sol-Gel Science and Technology, vol. 26, no. 1-3, pp. 915-918, 2003.

[2] T. M. Davis, M. A. Snyder, and M. Tsapatsis, "Germania nanoparticles and nanocrystals at room temperature in water and aqueous lysine sols," Langmuir, vol. 23, no. 25, pp. 1246912472, 2007.

[3] M. Sahnoun, C. Daul, R. Khenata, and H. Baltache, "Optical properties of germanium dioxide in the rutile structure," European Physical Journal B, vol. 45, no. 4, pp. 455-458, 2005.

[4] Z. Jiang, T. Xie, G. Z. Wang et al., " $\mathrm{GeO}_{2}$ nanotubes and nanorods synthesized by vapor phase reactions," Materials Letters, vol. 59, no. 4, pp. 416-419, 2005.

[5] L. Pajasová, "Optical properties of $\mathrm{GeO}_{2}$ in the ultraviolet region," Czechoslovak Journal of Physics, vol. 19, no. 10, pp. 1265-1270, 1969.

[6] A. Trukhin, M. Kink, Y. Maksimov, J. Jansons, and R. Kink, "Luminescence of $\mathrm{GeO}_{2}$ glass, rutile-like and $\alpha$-quartz-like crystals," Journal of Non-Crystalline Solids, vol. 352, no. 2, pp. 160-166, 2006.

[7] E. Ghobadi and J. A. Capobianco, "Crystal properties of $\alpha$ quartz type $\mathrm{GeO}_{2}$," Physical Chemistry Chemical Physics, vol. 2, no. 24, pp. 5761-5763, 2000.

[8] M. Micoulaut, L. Cormier, and G. S. Henderson, "The structure of amorphous, crystalline and liquid $\mathrm{GeO}_{2}$," Journal of Physics Condensed Matter, vol. 18, no. 45, pp. R753-R784, 2006.

[9] M. R. Khanlary, P. D. Townsend, and N. Gustavsson, "Luminescence of rutile-like $\mathrm{GeO}_{2}$ crystals," Radiation Effects and Defects in Solids, vol. 127, no. 1, pp. 61-74, 1993.

[10] H. P. Wu, J. F. Liu, M. Y. Ge et al., "Preparation of monodisperse $\mathrm{GeO}_{2}$ nanocubes in a reverse micelle system," Chemistry of Materials, vol. 18, no. 7, pp. 1817-1820, 2006.

[11] M. Zheng, M. Gu, Y. Jin, and G. Jin, "Preparation, structure and properties of $\mathrm{TiO}_{2}$-PVP hybrid films," Materials Science and Engineering B, vol. 77, no. 1, pp. 55-59, 2000. 
[12] A. A. Bol and A. Meijerink, "Doped semiconductor nanoparticles-a new class of luminescent materials?" Journal of Luminescence, vol. 87, pp. 315-318, 2000.

[13] S. J. Xu, X. C. Wang, S. J. Chua et al., "Effects of rapid thermal annealing on structure and luminescence of self-assembled InAs/GaAs quantum dots," Applied Physics Letters, vol. 72, no. 25, pp. 3335-3337, 1998.

[14] S. V. Manorama, K. Madhusudan Reddy, C. V. Gopal Reddy, S. Narayanan, P. Rajesh Raja, and P. R. Chatterji, "Photostabilization of dye on anatase titania nanoparticles by polymer capping," Journal of Physics and Chemistry of Solids, vol. 63, no. 1, pp. 135-143, 2002.

[15] N. Varghese, K. Biswas, and C. N. R. Rao, "Investigations of the growth kinetics of capped CdSe and CdS nanocrystals by a combined use of small angle X-ray scattering and other techniques," Chemistry, vol. 3, no. 8-9, pp. 1435-1442, 2008.

[16] Y.-W. Chiu and M. H. Huang, "Formation of Hexabranched $\mathrm{GeO}_{2}$ Nanoparticles via a Reverse Micelle System," Journal of Physical Chemistry C, vol. 113, no. 15, pp. 6056-6060, 2009.

[17] R. Fajgar, M. Jakoubková, Z. Bastl, and J. Pola, "Germaniumcontaining coatings by IR laser-induced decomposition of ethoxy(trimethyl) germane and tetramethylgermane," Applied Surface Science, vol. 86, no. 1-4, pp. 530-532, 1995.

[18] X. Feng, Y. Liu, C. Lu, W. Hou, and J.-J. Zhu, "Onestep synthesis of $\mathrm{AgCl} /$ polyaniline core-shell composites with enhanced electroactivity," Nanotechnology, vol. 17, no. 14, pp. 3578-3583, 2006.

[19] X. Lu, L. Li, W. Zhang, and C. Wang, "Preparation and characterization of $\mathrm{Ag}_{2} \mathrm{~S}$ nanoparticles embedded in polymer fibre matrices by electrospinning," Nanotechnology, vol. 16, no. 10, pp. 2233-2237, 2005.

[20] E. Hao and T. Lian, "Buildup of polymer/Au nanoparticle multilayer thin films based on hydrogen bonding," Chemistry of Materials, vol. 12, no. 11, pp. 3392-3396, 2000.

[21] M. Zawadzki and J. Okal, "Synthesis and structure characterization of $\mathrm{Ru}$ nanoparticles stabilized by PVP or $\gamma-\mathrm{Al}_{2} \mathrm{O}_{3}$," Materials Research Bulletin, vol. 43, no. 11, pp. 3111-3121, 2008.

[22] M. Sahnoun, C. Daul, R. Khenata, and H. Baltache, "Optical properties of germanium dioxide in the rutile structure," European Physical Journal B, vol. 45, no. 4, pp. 455-458, 2005.

[23] S. H. Liu, X. F. Qian, J. Yin, X. D. Ma, J. Y. Yuan, and Z. K. Zhu, "Preparation and characterization of polymer-capped CdS nanocrystals," Journal of Physics and Chemistry of Solids, vol. 64, no. 3, pp. 455-458, 2003. 

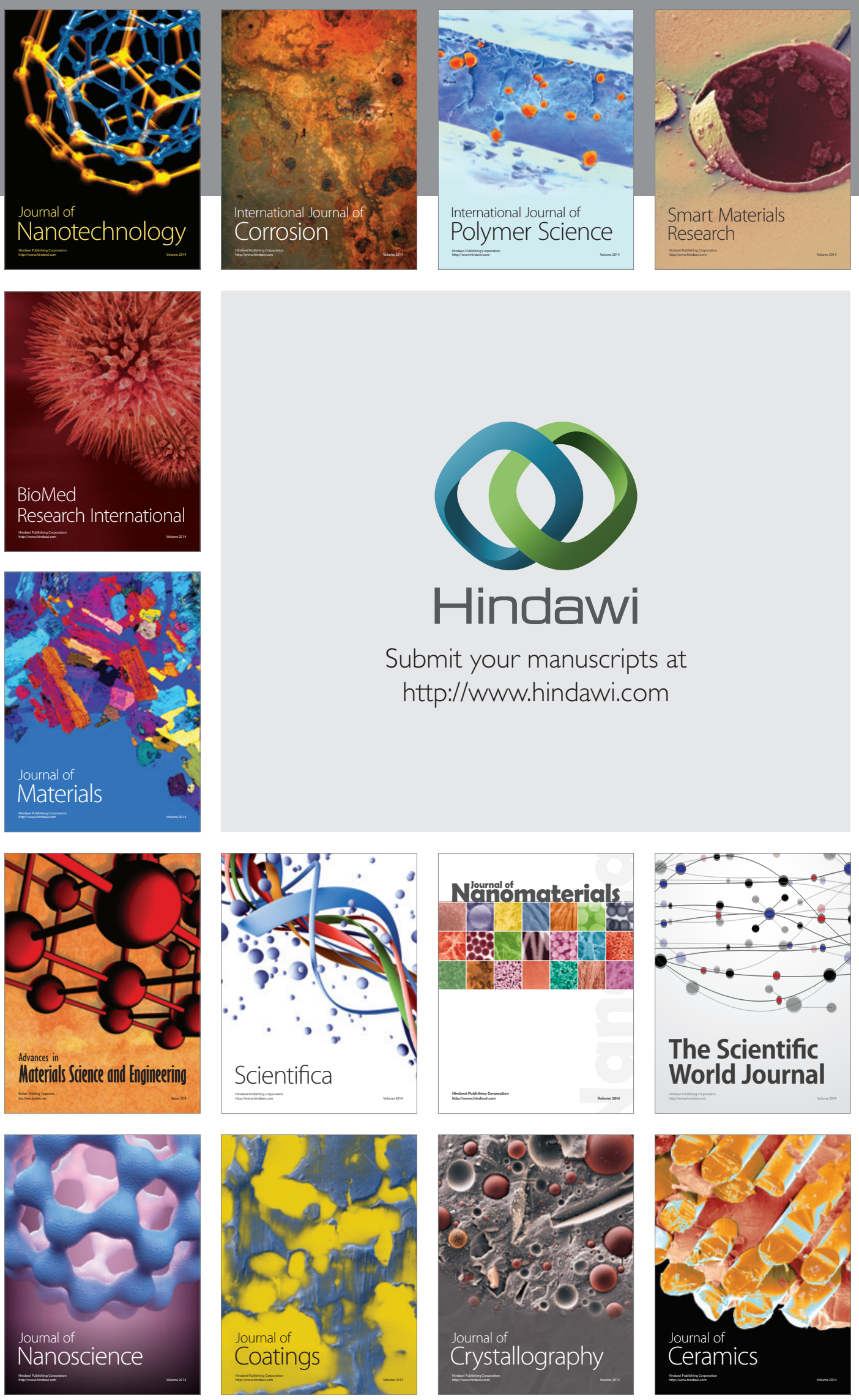

The Scientific World Journal

Submit your manuscripts at

http://www.hindawi.com

\section{World Journal}

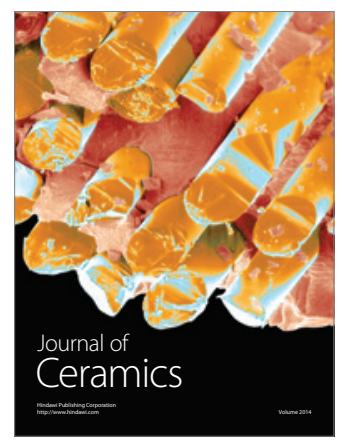

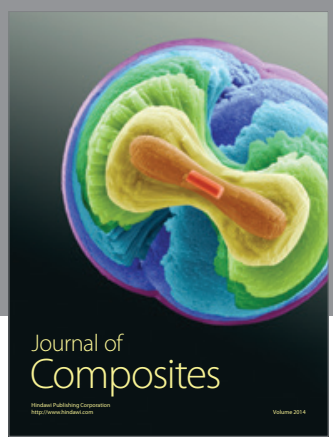
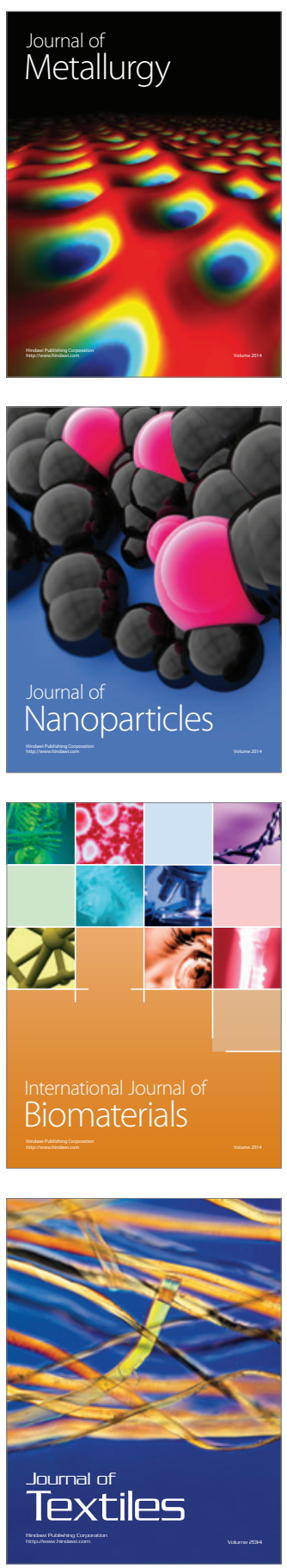